S. Goto

Nagoya Math. J.

Vol. 71 (1978), 1-12

\title{
THE RANK OF SYZYGIES UNDER THE ACTION BY A FINITE GROUP
}

\author{
SHIRO GOTO
}

\section{Introduction}

Let $S$ be a Noetherian local ring with maximal ideal $J$ and $k$ the residue field of $S$. Let $G$ be a finite group of order $n$ and suppose that $G$ acts on $S$ as automorphisms. Let $R=S^{G}$ and $I=J^{G}$. We denote by $S[G]$ (resp. $R[G]$ ) the twisted group ring of $G$ over $S$ (resp. the group algebra of $G$ over $R$ ). Recall that the multiplication of $S[G]$ is defined as follows: $s g \cdot t h=s g(t) \cdot g h$ for $s, t \in S$ and $g, h \in G$. Let $t_{G}(S)$ $=\left\{\sum_{g \in G} g(s) / s \in S\right\}$ and call it the trace ideal of $S$. Note that $t_{G}(S)=R$ if $n$ is a unit of $S$. We say that $S$ has a normal basis if $S \cong R[G]$ as $R[G]$-modules. This condition says that there is an element $s$ of $S$ so that $\{g(s)\}_{g \in G}$ forms an $R$-free basis of $S$.

Let $M$ be a finitely generated $S$-module and let $i \geqq 0$ be an integer. We put $\beta_{i}^{S}(M)=\operatorname{dim}_{k} \operatorname{Tor}_{i}^{S}(k, M)$ and call it the $i$-th rank of syzygies of $M$. It is well-known that, if $\cdots \rightarrow F_{i} \rightarrow \cdots \rightarrow F_{1} \rightarrow F_{0} \rightarrow M \rightarrow 0$ is a minimal free resolution of $M$, the number $\beta_{i}^{S}(M)$ is equal to the rank of $F_{i}$. The purpose of this paper is to find the relation between $\beta_{i}^{S}(M)$ and $\beta_{i}^{R}\left(M^{G}\right)$ for an $S[G]$-module $M$ (In this paper all $S[G]$-modules are assumed to be left modules.). The existence of normal bases will play an important role in this subject and this point of view was suggested to the author by Professor S. Endo.

The main result is

THEOREM (1.1). Suppose that $t_{G}(S)=R$ and let $H=\operatorname{Ker}(G \rightarrow$ Aut $k)$. Then the following two conditions are equivalent.

(1) $S$ has a normal basis relative to $H$.

(2) $S$ is a finitely generated free $R$-module of rank $n$, and the inequality $\beta_{i}^{S}(M) \geqq \beta_{i}^{R}\left(M^{G}\right)$ holds for every finitely generated $S[G]$-module $M$ and for every integer $i \geqq 0$.

Received January 28, 1977. 
In this case, if $n$ is a unit of $S$, then $S$ must have a normal basis relative to $G$.

When $S$ is an integral domain, we can improve this as follows:

COROLLARY (1.2). Suppose that $S$ is an integral domain and that $G$ is a subgroup of Aut $S$. Then the following conditions are equivalent.

(1) $S$ has a normal basis relative to $G$.

(2) $S$ is a free $R$-module and $t_{G}(S)=R$.

(3) Let $M$ be a finitely generated $S[G]-$ module. Then $M^{G}$ is a finitely generated $R$-module and $\beta_{0}^{S}(M) \geqq \beta_{0}^{R}\left(M^{G}\right)$.

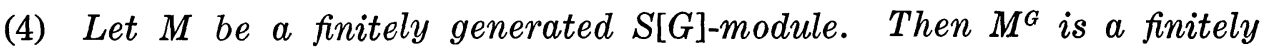
generated $R$-module and $\beta_{i}^{S}(M) \geqq \beta_{i}^{R}\left(M^{G}\right)$ for every integer $i \geqq 0$.

(1.2) is a supplement of the Chevalley-Serre theorem. More precisely, suppose that $S$ is an integral domain with $t_{G}(S)=R$ and that $G$ is a subgroup of Aut $S$. Then it can be shown that $S$ is a free $R$-module if $H$ is generated by generalized reflections on $S$ (i.e., such elements $g$ of Aut $S$ that $(s-g(s) / s \in S) \subset t S$ for some $t \in J)$. When $S$ is a regular local ring, the converse is also true (c.f. [2] and [7]). In these cases (1.2) is applicable (c.f. (5.2)).

If $G$ is a subgroup of Aut $S$, the conditions (1) and (2) of (1.2) are equivalent for an arbitrary (i.e., not necessarily Noetherian) local domain $S$. Therefore, in case $S$ is an integral domain and $t_{G}(S)=R, S$ has a normal basis relative to $G$ if and only if $S$ has a normal basis relative to $H$ (c.f. (5.1)). (Of course this is not true in case $S$ is not an integral domain. Counterexamples are easily given.)

In Section 2 we give a few remarks on the ideal $t_{G}(S)$ and the functor []$^{G}$, which we shall need later. In Section 3 we will show that the equality $\beta_{i}^{S}(M)=\beta_{i}^{R}\left(M^{G}\right)$ holds for every $S[G]$-module $M$ and for every integer $i \geqq 0$ if $G$ acts on $k$ faithfully. In Section 4 (resp. Section 5) we prove Theorem (1.1) (resp. Corollary (1.2)). In Section 6 we will give some consequences of Theorem (1.1), from which the motivation for this research has come.

\section{The exactness of the functor []$^{G}$}

In this section $S$ need not be Noetherian.

LEMMA (2.1). $S[G]^{G}=d S$ where $d=\sum_{g \in G} g . \quad$ The map $f: S \rightarrow S[G]^{G}$, $f(s)=d s$ for $s \in S$, is an R-isomorphism. 
Proof. Of course $d s=\sum_{g \in G} g(s) g$ is invariant for every $s \in S$. Let $a=\sum_{g \in G} a_{g} g$ be an element of $S[G]^{G}\left(a_{g} \in S\right)$. Then, as $g a=a$, we have $a_{g h}=g\left(a_{h}\right)$ for all $g, h \in G$. Hence $a_{g}=g\left(a_{1}\right)$ and so $a=d s$ for $s=a_{1}$. The second assertion is trivial.

We put $t_{G}(M)=\left\{\sum_{g \in G} g(m) / m \in M\right\}$ for an $S[G]$-module $M . \quad t_{G}(M)$ is an $R$-submodule of $M^{G}$ and the operator $t_{G}: M \rightarrow M^{G}, t_{G}(m)=\sum_{g \in G} g(m)$, is an $R$-homomorphism.

Proposition (2.2). The following conditions are equivalent.

(1) []$^{G}$ is exact.

(2) $t_{G}(S)=R$.

(3) $S$ is a (finitely generated) projective $S[G]$-module.

(4) $t_{G}: M \rightarrow M^{G}$ is a (split) $R$-epimorphism for every $S[G]$-module $M$.

(5) Let $M$ be an S[G]-module. Then $M$ is a projective S[G]-module if (and only if) $M$ is a free $S$-module.

(6) Let $E: 0 \rightarrow M^{\prime} \rightarrow M \rightarrow M^{\prime \prime} \rightarrow 0$ be an exact sequence of $S[G]$-modules. Then $E$ splits as a sequence of $S[G]$-modules if (and only if) it splits as a sequence of $S$-modules.

Proof. (1) $\Rightarrow(2)$ Let $f: S[G] \rightarrow S$ be the $S[G]$-epimorphism given by $f(a)=a 1$ for all $a \in S[G]$. Then $f\left(S[G]^{G}\right)=R$ by the assumption. Hence $t_{G}(S)=R$, as $f\left(S[G]^{G}\right)=t_{G}(S)$ (c.f. (2.1)).

(2) $\Rightarrow$ (4) Let $M$ be an $S[G]$-module and let $s \in S$ such that $\sum_{g \in G} g(s)$ $=1$. We define a map $f: M^{G} \rightarrow M$ by $f(x)=s x$ for all $x \in M^{G}$. Then $t_{G} \circ f=1_{M^{G}}$ as $\sum_{g \in G} g(s)=1$. Hence $t_{G}$ is a split $R$-epimorphism.

$(4) \Rightarrow(1)$ Since the functor [ $]^{G}$ is left exact in general, it suffices to show that [ ] ${ }^{G}$ preserves epimorphisms. Now let $f: M \rightarrow N$ be an $S[G]$-epimorphism. Then $M^{G}=t_{G}(M)$ and $N^{G}=t_{G}(N)$ by the assumption. Hence, as $f$ is compatible with $t_{G}$, we have $f\left(M^{G}\right)=N^{G}$.

$(2) \Rightarrow(6)$ Let $s \in S$ such that $\sum_{g \in G} g(s)=1$. Let $E: 0 \rightarrow M^{\prime} \rightarrow M$ $\stackrel{f}{\longrightarrow} M^{\prime \prime} \rightarrow 0$ be an exact sequence of $S[G]$-modules and assume that $E$ splits as a sequence of $S$-modules. We choose an $S$-linear map $f^{\prime}: M^{\prime \prime} \rightarrow M$ so that $f \circ f^{\prime}=1_{M \prime \prime}$. Now let $f^{\prime \prime}=\sum_{g \in G} r_{M}(g) \circ s f^{\prime} \circ r_{M^{\prime \prime}}\left(g^{-1}\right) \quad$ where $r_{M}(g)$ (resp. $r_{M^{\prime \prime}}\left(g^{-1}\right)$ ) denotes the action of $g$ (resp. $g^{-1}$ ) on $M$ (resp. $M^{\prime \prime}$ ). Then a direct computation shows that the $S$-linear map $f^{\prime \prime}: M^{\prime \prime} \rightarrow M$ is compatible with $G$-action and that $f \circ f^{\prime \prime}=1_{M^{\prime \prime}}$.

(6) $\Rightarrow(5) \Rightarrow(3)$ This is trivial.

$(3) \Rightarrow(2) \quad$ The $S[G]$-epimorphism $f: S[G] \rightarrow S$ defined by $f(a)=a 1$ 
for all $a \in S[G]$ splits by the assumption. Hence $f\left(S[G]^{G}\right)=R$, and so we have $t_{G}(S)=R$ as $f\left(S[G]^{G}\right)=t_{G}(S)$ by $(2.1)$.

\section{The case where $G$ acts on $k$ faithfully}

In this section $S$ need not be Noetherian. We assume that $G$ is a subgroup of Aut $S$.

Lemma (3.1). Suppose that $S$ is a field. Then there exists an $S$ basis of $S[G]$ consisting of invariants.

Proof. By the Galois theory of fields, $S$ must have a normal basis. Let $s \in S$ such that $\{g(s)\}_{g \in G}$ is an $R$-basis of $S$. Then the matrix $[(g h)(s)]_{g, h \in G}$ is invertible and so $\left\{\sum_{g \in G}(g h)(s) g\right\}_{h \in G}$ forms an $S$-basis of $S[G]$. Of course these elements are $G$-invariant.

Proposition (3.2). The following conditions are equivalent.

(1) $G$ acts on $k$ faithfully, i.e., $\operatorname{Ker}(G \rightarrow$ Aut $k)=1$.

(2) $S$ is a finitely generated $R$-module, $t_{G}(S)=R$, and $J=I S$.

(3) $S$ has a normal basis relative to $G$ and $J=I S$.

In this case $S[G]$ has an $S$-free basis consisting of invariants.

Proof. Suppose (1) and we will prove the last assertion. First notice that $t_{G}(S)=R$. In fact, $t_{G}(k) \neq(0)$ as the field $k$ has a normal basis relative to $G$, and so $t_{G}(S) \not \subset I$. Now let $f: S[G] \rightarrow k[G]$ $=S[G] / J S[G]$ be the canonical epimorphism. Then we have $f\left(S[G]^{G}\right)$ $=k[G]^{G}$ since the functor []$^{G}$ is exact by (2.2). Hence there is a family $\left\{e_{i}\right\}_{1 \leqq i \leqq n}$ of elements of $S[G]^{G}$ such that $k[G]=\sum_{i=1}^{n} k f\left(e_{i}\right)$, because $k[G]$ has a $k$-basis consisting of invariants (c.f. (3.1)). Thus we have $S[G]$ $=\sum_{i=1}^{n} S e_{i}$ by Nakayama's lemma. Notice that $\left\{e_{i}\right\}_{1 \leqq i \leqq n}$ are linearly independent over $S$ as $S[G]$ is a finitely generated free $S$-module of rank $n$.

$(1) \Rightarrow(3) \quad$ We have $S[G]^{G}=\sum_{i=1}^{n} R e_{i}$ as $S[G]^{G}=t_{G}(S[G])$ by (2.2). Therefore $S$ is a finitely generated free $R$-module of rank $n$, since $S \cong S[G]^{G}$ as $R$-modules by (2.1). In particular $\operatorname{dim}_{R / I} S / I S=n$. On the other hand, since the field extension $S / J$ over $R / I$ is finite and Galois with Galois group $G$, we have $\operatorname{dim}_{R / I} S / J=n$. Thus $J=I S$ as $\operatorname{dim}_{R / I} S / J$ $=\operatorname{dim}_{R / I} S / I S$. Now let us prove that $S \cong R[G]$ as $R[G]$-modules. For this purpose we have only to show that $S$ is a cyclic $R[G]$-module, because $S$ and $R[G]$ are free $R$-modules of the common rank $n$. By 
Nakayama's lemma it suffices to prove that $S / I S$ is cyclic as an $(R / I)[G]$ module. But this is obvious since $J=I S$ and since $S / J$ has a normal basis (Recall $(S / I S)^{G}=R / I$.).

(3) $\Rightarrow(2)$ This is trivial.

(2) $\Rightarrow$ (1) We put $H=\operatorname{Ker}(G \rightarrow$ Aut $k)$. Then $t_{H}(S)=S^{H}$ and, as the functor [ $]^{H}$ is exact by (2.2), we have $S^{H} / J^{H}=S / J$. On the other hand $J=J^{H} S$ as $J=I S$ by the assumption. Therefore $S=S^{H}+J^{H} S$. Hence we have $S=S^{H}$ by Nakayama's lemma and this completes the proof.

CoRollary (3.3). Suppose that $G$ acts on $k$ faithfully. Then the equality $\beta_{i}^{S}(M)=\beta_{i}^{R}\left(M^{G}\right)$ holds for every $S[G]$-module $M$ and for every integer $i \geqq 0$.

Proof. Let $N$ be an $R$-module. We regard $S \otimes_{R} N$ as an $S[G]$-module by the following G-action: $g(s \otimes x)=g(s) \otimes x$ for $g \in G, s \in S$, and $x \in N$. Let $f_{N}: N \rightarrow\left[S \otimes_{R} N\right]^{G}$ be the $R$-linear map defined by $f_{N}(x)=1 \otimes x$ for all $x \in N$. Conversely let $M$ be an $S[G]$-module and consider $S \otimes_{R} M^{G}$ to be an $S[G]$-module as above. Then, since $M^{G}$ is an $R$-submodule of $M$, there is an $S[G]$-linear map $g_{M}: S \otimes_{R} M^{G} \rightarrow M$ such that $g_{M}(1 \otimes x)=x$ for all $x \in M^{G}$. To prove the assertion it suffices to show that $f_{N}$ and $g_{M}$ are isomorphisms for every $R$-module $N$ and for every $S[G]$-module $M$. Now consider $f_{N}$. It is a monomorphism, as $S$ is a free $R$-module by (3.2). On the other hand $\left[S \otimes_{R} N\right]^{G}=\operatorname{Im} f_{N}$, since $\left[S \otimes_{R} N\right]^{G}$ $=t_{G}\left(S \otimes_{R} N\right)$ by (2.2). Thus $f_{N}$ is an isomorphism.

Let us consider $g_{M}$ and suppose that $F_{1} \rightarrow F_{0} \rightarrow M \rightarrow 0$ is a presentation of $M$ by free $S[G]$-modules $F_{i}$. Then, as [ $]^{G}$ is exact, we have a commutative diagram

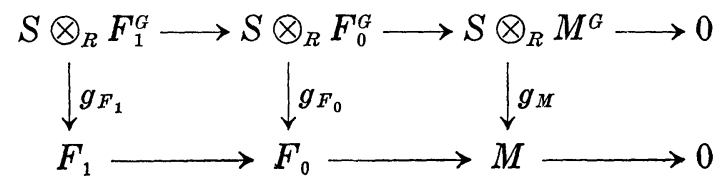

of $S[G]$-modules with exact rows. Hence to show that $g_{M}$ is an isomorphism we may assume that $M=S[G]$. In this case it is an epimorphism by the last assertion of (3.2). Thus we have verified that $g_{S[G]}$ is an isomorphism, since both of the $S$-modules $S[G]$ and $S \otimes_{R} S[G]^{G}$ are finitely generated and free of rank $n$. 


\section{Proof of Theorem (1.1)}

Proof of Theorem (1.1). (1) $\Rightarrow(2)$ First notice that $t_{H}(S)=S^{H}$ by (2.2), as $t_{G}(S)=R$. Then we have $S^{H} / J^{H}=S / J$, since the functor [ $]^{H}$ is exact. Hence the group $G / H$ acts on the residue field $S^{H} / J^{H}=S / J$ of $S^{H}$ faithfully. Therefore we have by (3.2) that $S^{H}$ is a finitely generated free $R$-module of $\operatorname{rank}|G / H|$. Thus $S$ is a finitely generated free $R$-module of rank $n$, as $S$ is a finitely generated free $S^{H}$-module of rank $|H|$ by the assumption. Now let $M$ be a finitely generated $S[G]$-module. Then, by (3.3), we have $\beta_{i}^{S^{H}}\left(M^{H}\right)=\beta_{i}^{R}\left(M^{G}\right)$ for every integer $i \geqq 0$. Therefore, to show that (1) implies (2), it suffices to prove in case $H=G$. Moreover we may assume that $R$ is complete.

We put $\bar{M}=M / J M$. Notice that $k[G]$ is a semisimple ring, since $n$ is a unit of $S$. Therefore, as $R$ is complete and as $k=R / I$, we can find a finitely generated projective $R[G]$-module $L$ so that $L / I L=\bar{M}$ as $k[G]$-modules (c.f. Proposition 2.12, p. 90, [1]). Now let $f: L \rightarrow \bar{M}$ be the epimorphism of $R[G]$-modules which is induced by the isomorphism $L / I L=\bar{M}$ and the canonical map $L \rightarrow L / I L$. We put $F=S \otimes_{R} L$ and consider it to be an $S[G]$-module by the following $G$-action: $g(s \otimes x)$ $=g(s) \otimes g(x)$ for $g \in G, s \in S$, and $x \in L$. Then, since $\bar{M}$ is an $S[G]-$ module, the epimorphism $f: L \rightarrow \bar{M}$ of $R[G]$-modules can be extended to an epimorphism $f^{\prime}: F=S \otimes_{R} L \rightarrow \bar{M}$ of $S[G]$-modules. Notice that $F$ is a finitely generated free $S$-module as $L$ is a finitely generated free $R$-module. Then, by (2.2), $F$ is a finitely generated projective $S[G]-$ module. Thus the epimorphism $f^{\prime}: F \rightarrow \bar{M}$ is factorized as follows

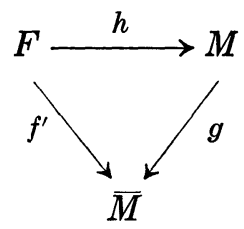

where $g: M \rightarrow \bar{M}$ denotes the canonical epimorphism. Notice that $h$ is an epimorphism by Nakayama's lemma.

Now let $\left\{e_{i}\right\}_{1 \leqq i \leqq r}$ be an $R$-free basis of $L(r=\operatorname{rank} L)$. Since $F^{G}$ $=t_{G}(F)$ by (2.2), we have that $F^{G}=\sum_{i=1}^{r} R t_{G}\left(s \otimes e_{i}\right)$ where $s$ is an element of $S$ such that $\{g(s)\}_{g \in G}$ forms an $R$-free basis of $S$. Thus we have known that the rank of the finitely generated free $R$-module $F^{G}$ is at most $r$. Therefore, as [ $]^{G}$ is exact, we have that $M^{G}$ is a finitely gen- 
erated $R$-module with $\beta_{0}^{R}\left(M^{G}\right) \leqq r$. Notice that $r=\beta_{0}^{S}(M)$, as $r=\operatorname{dim}_{k} \bar{M}$. Then we have $\beta_{0}^{S}(M) \geqq \beta_{0}^{R}\left(M^{G}\right)$. Repeating the argument above we shall have that $\beta_{i}^{S}(M) \geqq \beta_{i}^{R}\left(M^{G}\right)$ for every integer $i \geqq 0$. Thus we complete the proof of the assertion (1) $\Rightarrow(2)$.

LEMMA (4.1). Suppose that $t_{G}(S)=R$ and let $H=\operatorname{Ker}(G \rightarrow$ Aut $k)$. If $M^{G}$ is a finitely generated $R$-module with $\beta_{0}^{R}\left(M^{G}\right) \leqq \beta_{0}^{S}(M)$ for every finitely generated $S[G]-m o d u l e ~ M$, then $S$ is a cyclic $S^{H}[H]$-module. In particular, if $n$ is a unit of $S$, then $S$ is cyclic as an $R[G]-$ module.

Proof. First we discuss in case $n$ is a unit of $S$. By the assumption $S[G]^{G}$ is a finitely generated $R$-module. Therefore so is $S$, since $S \cong S[G]^{G}$ as $R$-modules (c.f. (2.1)). Hence, to show that $S$ is cyclic as an $R[G]$-module, it suffices to prove that $S / I S$ is a cyclic $(R / I)[G]-$ module. Therefore we may assume without loss of generality that $R$ is a field. In this situation, $R[G]$ is a semisimple ring since $n$ is a unit of $S$. Now assume the contrary. Then there is a simple $R[G]$-module $V$ such that $(m+1) V$ is a direct summand of $S$ as an $R[G]$-module, where $m$ denotes the multiplicity of $V$ in $R[G]$. We put ${ }^{t} V=\operatorname{Hom}_{R}(V, R)$ and regard it as an $R[G]$-module by the following $G$-action : $g(f)=f \circ g^{-1}$ for $g \in G$ and $f \in \operatorname{Hom}_{R}(V, R)$. Now consider the $S[G]$-module $M=$ $S \otimes_{R}{ }^{t} V$. Of course $M$ is a finitely generated $S$-module with $\beta_{0}^{S}(M)$ $=\operatorname{dim}_{R} V$. Hence we have $\beta_{0}^{S}(M)=\operatorname{dim}_{R} V \geqq \beta_{0}^{R}\left(M^{G}\right)=\operatorname{dim}_{R} M^{G}$ by the assumption. On the other hand, since $M$ contains $(m+1)\left(V \otimes_{R}{ }^{t} V\right)$ as an $R[G]$-submodule, we see that $\operatorname{dim}_{R} M^{G} \geqq(m+1) \cdot \operatorname{dim}_{R}\left(V \otimes_{R}{ }^{t} V\right)^{G}$. Therefore we have that $\operatorname{dim}_{R} M^{G}>m \cdot \operatorname{dim}_{R} \operatorname{End}_{R[G]} V$, since $\operatorname{dim}_{R}\left(V \otimes_{R}{ }^{t} V\right)^{G}$ $=\operatorname{dim}_{R} \operatorname{End}_{R[G]} V$ (c.f. (43.14) Theorem, [3]). Thus we conclude that $\operatorname{dim}_{R} M^{G}>\operatorname{dim}_{R} V$ by virtue of the well-known fact $\operatorname{dim}_{R} V=m$. $\operatorname{dim}_{R} \operatorname{End}_{R[G]} V$. This is a contradiction, and we have verified that $S$ is a cyclic $R[G]$-module.

Now back to the proof of the first assertion. As $|H|$ is a unit of $S$ it suffices by the second assertion to show that $N^{H}$ is a finitely generated $S^{H}$-module with $\beta_{0}^{S^{H}}\left(N^{H}\right) \leqq \beta_{0}^{S}(N)$ for every finitely generated $S[H]$-module $N$. Let $\left\{g_{i}\right\}_{1 \leqq i \leqq r}(r=|G / H|)$ be a system of representatives of $G \bmod H$. Then a straightforward computation shows that $t_{G}\left(S[G] \otimes_{S[H]} N\right)=\left\{\sum_{i=1}^{r} g_{i} \otimes t_{H}(x) / x \in N\right\}$. Hence, as $t_{G}(S)=R$ and as $t_{H}(S)=S^{H}$, we have $\left(S[G] \otimes_{S[H]} N\right)^{G}=\left\{\sum_{i=1}^{r} g_{i} \otimes x / x \in N^{H}\right\}$. Therefore we have that $\left(S[G] \otimes_{S[H]} N\right)^{G} \cong N^{H}$ as $R$-modules. Thus, by the assump- 
tion, we conclude that $N^{H}$ is a finitely generated $R$-module with $\beta_{0}^{R}\left(N^{H}\right)$ $=\beta_{0}^{R}\left(\left(S[G] \otimes_{S[H]} N\right)^{G}\right) \leqq \beta_{0}^{S}\left(S[G] \otimes_{S[H]} N\right)$. Of course $N^{H}$ is a finitely generated $S^{H}$-module. Now let $N_{i}=\left\{g_{i} \otimes x / x \in N\right\}$ in $S[G] \otimes_{S[H]} N(1 \leqq i \leqq r)$. Then $N_{i}$ is an $S$-submodule of $S[G] \otimes_{S[H]} N$ and $S[G] \otimes_{S[H]} N=\bigoplus_{i=1}^{r} N_{i}$ as $S$-modules. Hence, as $N_{i}$ is a finitely generated $S$-module with $\beta_{0}^{S}\left(N_{i}\right)$ $=\beta_{0}^{S}(N)$, we have that $\beta_{0}^{S}\left(S[G] \otimes_{S[H]} N\right)=r \cdot \beta_{0}^{S}(N)$. Summarizing the above results we see

$$
\begin{aligned}
\beta_{0}^{S}(N) & =1 / r \cdot \beta_{0}^{S}\left(S[G] \otimes_{S[H]} N\right) \\
& \geqq 1 / r \cdot \beta_{0}^{R}\left(\left(S[G] \otimes_{S[H]} N\right)^{G}\right) \\
& =1 / r \cdot \beta_{0}^{R}\left(N^{H}\right) .
\end{aligned}
$$

On the other hand, we have that $1 / r \cdot \beta_{0}^{R}\left(N^{H}\right)=\beta_{0}^{S^{H}}\left(N^{H}\right)$, as $\operatorname{dim}_{R / I} S^{H} / J^{H}$ $=r$ by (3.2). Thus we conclude that $\beta_{0}^{S}(N) \geqq \beta_{0}^{S^{H}}\left(N^{H}\right)$ as desired.

Proof of Theorem (1.1) (continued). First we prove that (2) implies the last assertion. If $n$ is a unit of $S, S$ is a cyclic $R[G]$-module by (4.1). This implies that $S \cong R[G]$ as $R[G]$-modules, because $S$ and $R[G]$ are finitely generated free $R$-modules of rank $n$.

(2) $\Rightarrow$ (1) As $S$ is a cyclic $S^{H}[H]$-module by (4.1), it suffices to show that $S$ is a finitely generated free $S^{H}$-module of rank $|H|$. Now consider the equality $\operatorname{dim}_{R / I} S / I S=\operatorname{dim}_{R / I} S^{H} / J^{H} \cdot \operatorname{dim}_{S^{H} / J^{H}} S / I S$, which follows from the fact $J^{H}=I S^{H}$ (c.f. (3.2)). Then, as $\operatorname{dim}_{R / I} S^{H} / J^{H}=|G / H|$ by (3.2) and as $\operatorname{dim}_{R / I} S / I S=n$ by the assumption, we have $\operatorname{dim}_{S^{H} / J^{H}} S / J^{H} S$ $=|H|$. Hence $S$ is a finitely generated $S^{H}$-module with $\beta_{0}^{S^{H}}(S)=|H|$. This implies that $S$ is a free $S^{H}$-module, since $S$ is a finitely generated free $R$-module of rank $n$ by the assumption and since $S^{H}$ is a finitely generated free $R$-module of $\operatorname{rank}|G / H|$ by (3.2). Thus we have verified the assertions of Theorem (1.1).

Remark (4.2). Suppose that $n$ is a unit of $S$ and let $H=$ $\operatorname{Ker}(G \rightarrow$ Aut $k)$. If $S$ has a normal basis relative to $H$, then $S$ has a normal basis relative to $G$.

\section{Proof of Corollary (1.2)}

Proposition (5.1). Suppose that $S$ is a (not necessarily Noetherian) local domain and that $G$ is a subgroup of Aut $S$. Let $H=\operatorname{Ker}(G \rightarrow$ Aut $k)$. Then the following conditions are equivalent.

(1) $S$ has a normal basis relative to $G$. 
(2) $S$ has a norma! basis relative to $H$ and $t_{G}(S)=R$.

(3) $S$ is a free $R$-module and $t_{G}(S)=R$.

Proof. (1) $\Rightarrow$ (3) This follows easily from (2.2).

(3) $\Rightarrow$ (1) Let $L$ (resp. $K$ ) be the quotient field of $S$ (resp. $R$ ). Then the field extension $L / K$ is finite and $L=K \otimes_{R} S$. Therefore $S$ is a finitely generated $R$-module. Hence $S$ is a finitely generated projective $R[G]$-module, as $S$ is projective as an $S[G]$-module by (2.2) and as $S[G]$ is a free $R[G]$-module. Thus, to prove $S \cong R[G]$ as $R[G]$-modules, it suffices to show that $L \cong K[G]$ as $K[G]$-modules (c.f. Proposition 5.1, p. $590,[1])$, and this follows from the Galois theory of fields.

$(2) \Rightarrow(1) \quad B y$ the assumption $S$ is a free $S^{H}$-module. On the other hand $S^{H}$ is a free $R$-module by (3.2). Hence $S$ is a free $R$-module with $t_{G}(S)=R$.

(1) $\Rightarrow$ (2) It follows from (2.2) that $t_{G}(S)=R$ and so we have $t_{H}(S)$ $=S^{H}$. Thus, by virtue of the equivalence of (1) and (3), we have only to prove that $S$ is a free $S^{H}$-module. Consider the equality $\operatorname{dim}_{R / I} S^{H} / J^{H}$. $\operatorname{dim}_{S^{H} / J^{H}} S / I S=\operatorname{dim}_{R / I} S / I S$, which follows from the fact $J^{H}=I S^{H}$ (c.f. (3.2)). Then we have $\operatorname{dim}_{S^{H} / J^{H}} S / J^{H} S=|H|$. Let $K^{\prime}$ be the quotient field of $S^{H}$. Then $\left[L: K^{\prime}\right]=|H|$ and $L=K^{\prime} \otimes_{S^{H}} S$. Hence $S$ is a free $S^{H_{-}}$ module of $\operatorname{rank}|H|$ as $\beta_{0}^{S^{H}}(S)=|H|$.

Proof of Corollary (1.2). (1) $\Leftrightarrow$ (2) This is proved by (5.1).

(1) $\Rightarrow$ (4) This follows from (5.1) and (1.1).

(4) $\Rightarrow(3)$ This is trivial.

(3) $\Rightarrow(2) \quad$ By the assumption $S[G]^{G}$ is a finitely generated $R$-module with $\beta_{0}^{R}\left(S[G]^{G}\right) \leqq n$. Thus so is $S$, since $S \cong S[G]^{G}$ as $R$-modules. Recall that the field extension $L / K$ is finite of degree $n$ and that $L=K \otimes_{R} S$, where $L$ (resp. $K$ ) denotes the quotient field of $S$ (resp. $R$ ). Thus we have that $S$ is a free $R$-module of rank $n$. Now let us prove that $t_{G}(S)$ $=R$. Notice that $(S / I S)^{G}=R / I$, because $\beta_{0}^{R}\left((S / I S)^{G}\right) \leqq \beta_{0}^{S}(S / I S)=1$ by the assumption. Then, to show that $t_{G}(S)=R$, it suffices to prove that $t_{G}(S / I S) \neq(0)$. Consider the exact sequence

$$
0 \longrightarrow K \longrightarrow(S / I S)[G] \stackrel{f}{\longrightarrow} S / I S \longrightarrow 0
$$

where $f:(S / I S)[G] \rightarrow S / I S$ denotes the epimorphism given by $f(a)=a 1$ for all $a \in(S / I S)[G]$. Then we have an exact sequence 


$$
0 \longrightarrow K^{G} \longrightarrow((S / I S)[G])^{G} \stackrel{f}{\longrightarrow}(S / I S)^{G}=R / I
$$

of $R / I$-modules. Recall that $f\left(((S / I S)[G])^{G}\right)=t_{G}(S / I S)$ (c.f. (2.1)). Now assume that $t_{G}(S / I S)=(0)$. Then we have $K^{G}=((S / I S)[G])^{G}$, and so $\beta_{0}^{R}\left(K^{G}\right)=n$ by (2.1). On the other hand, as the sequence $\left(^{*}\right)$ is a split exact sequence of $S / I S$-modules, we have that $\beta_{0}^{S}(K)=n-1$. Therefore $\beta_{0}^{S}(K)=n-1<\beta_{0}^{R}\left(K^{G}\right)=n$-this is a contradiction. Thus we have $t_{G}(S / I S) \neq(0)$, and so $t_{G}(S)=R$. This completes the proof of Corollary (1.2).

Summarizing with the Chevalley-Serre theorem (c.f. [2] and [7]), we have

THEOREM (5.2). Suppose that $S$ is a regular local ring and that $G$ is a subgroup of Aut $S$. Let $H=\operatorname{Ker}(G \rightarrow$ Aut $k)$. Then the following conditions are equivalent.

(1) $t_{G}(S)=R$, and $H$ is generated by generalized reflections.

(2) $R$ is a regular local ring and $t_{G}(S)=R$.

(3) $S$ is a free $R$-module and $t_{G}(S)=R$.

(4) $S$ has a normal basis relative to $G$.

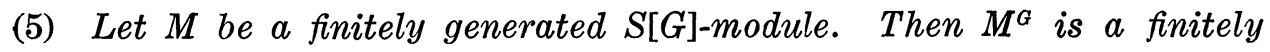
generated $R$-module and the inequality $\beta_{i}^{S}(M) \geqq \beta_{i}^{R}\left(M^{G}\right)$ holds for every integer $i \geqq 0$.

\section{Application}

In this section suppose that $S$ is a Noetherian local ring with $t_{G}(S)=R$ and that $S$ has a normal basis relative to $H=\operatorname{Ker}(G \rightarrow$ Aut $k)$.

Now recall some definitions. For the moment let $(A, m)$ be a Noetherian local ring and let $M$ be a finitely generated $A$-module. Then $M$ is said to be a Macaulay $A$-module if $\operatorname{dim}_{A} M=\operatorname{depth}_{A} M$. In this case we put $r_{A}(M)=\operatorname{dim}_{A / m} \operatorname{Ext}_{A}^{d}(A / m, M)\left(d=\operatorname{dim}_{A} M\right)$ and call it the type of $M$. Various properties of the invariant $r_{A}(M)$ are discussed in [6]. By definition the ring $A$ is a Gorenstein local ring if $A$ is a Macaulay local ring and $r(A)=1$. Let $\mathrm{hd}_{A} M$ denote the homological dimension of $M$ and let grade $_{A} M$ be the common length of maximal $A$ regular sequences contained in the annihilator of $M$. Recall that $\operatorname{grade}_{A} M=\inf \left\{i \in Z / \operatorname{Ext}_{A}^{i}(M, A) \neq(0)\right\}$. Hence $\operatorname{hd}_{A} M \geqq \operatorname{grade}_{A} M$ in general. $M$ is called a perfect $A$-module if $\mathrm{hd}_{A} M=\operatorname{grade}_{A} M$. A proper 
ideal $\underline{a}$ of $A$ is said to be perfect if $A / \underline{a}$ is a perfect $A$-module.

THEOREM (6.1). Let $M$ be a finitely generated $S[G]$-module and assume that $M^{G} \neq(0)$. Then

(1) $\mathrm{hd}_{S} M \geqq \mathrm{hd}_{R} M^{G}$.

(2) If $M$ is a Macaulay $S$-module, then $M^{G}$ is again a Macaulay $R$-module and $\operatorname{dim}_{R} M^{G}=\operatorname{dim}_{S} M$.

(3) Suppose that $S$ is a Macaulay local ring. If $M$ is a perfect $S$-module, then $M^{G}$ is again a perfect $R$-module and grade $_{R} M^{G}=\operatorname{grade}_{S} M . \quad$ In particular $r_{S}(M) \geqq r_{R}\left(M^{G}\right)$.

Proof. (1) This follows immediately from (1.1).

(2) Note that $M^{G}$ is a direct summand of $M$ as an $R$-module. Thus $M^{G}$ is a Macaulay $R$-module, because so is $M$ as an $R$-module. Of course $\operatorname{dim}_{R} M^{G}=\operatorname{dim}_{R} M=\operatorname{dim}_{S} M$.

(3) $M^{G}$ is a Macaulay $R$-module with $\operatorname{dim}_{R} M^{G}=\operatorname{dim}_{S} M$ by (2), since $M$ is a Macaulay $S$-module. Thus, as $\operatorname{dim} R=\operatorname{dim} S$, we have

$$
\begin{aligned}
\operatorname{hd}_{R} M^{G} & \geqq \operatorname{grade}_{R} M^{G} \\
& =\operatorname{dim} R-\operatorname{dim}_{R} M^{G} \\
& =\operatorname{dim} S-\operatorname{dim}_{S} M \\
& =\operatorname{grade}_{S} M \\
& =\operatorname{hd}_{S} M .
\end{aligned}
$$

Hence $\mathrm{hd}_{R} M^{G}=\operatorname{grade}_{R} M^{G}$ as $\mathrm{hd}_{S} M \geqq \mathrm{hd}_{R} M^{G}$ by (1). Therefore $M^{G}$ is a perfect $R$-module and $\operatorname{grade}_{R} M^{G}=\operatorname{grade}_{S} M$. For the second assertion notice that $r_{S}(M)=\beta_{d}^{S}(M) \cdot r(S)$ and that $r_{R}\left(M^{G}\right)=\beta_{d}^{R}\left(M^{G}\right) \cdot r(R)$ where $d=\operatorname{dim}_{S} M=\operatorname{dim}_{R} M^{G}$. These equalities are proved similarly as Proposition 2.1 of [4]. On the other hand, since $S$ is a flat $R$-module, we see $r(S) \geqq r(R)$ (c.f. Satz 1.24, [6]). Hence we have $r_{S}(M) \geqq r_{R}\left(M^{G}\right)$ as $\beta_{d}^{S}(M) \geqq \beta_{d}^{R}\left(M^{G}\right)$.

CoRollary (6.2) (c.f. [5]). Suppose that $S$ is a Macaulay local ring and let $\underline{b}$ be a perfect ideal of $S$. Assume that $\underline{b}$ is G-stable. Then $r(S / \underline{b}) \geqq r(R / \underline{a})$ where $\underline{a}=\underline{b}^{G}$. In particular, if $S / \underline{b}$ is a Gorenstein local ring, then so is the ring $R / \underline{a}$.

COROLlaRY (6.3) (c.f. [5]). Suppose that $S$ is a Macaulay local ring and let $\underline{b}$ be a G-stable ideal of $S$. If $\underline{b}$ is generated by an S-regular sequence, then $\underline{a}=\underline{b}^{G}$ is again generated by an $R$-regular sequence. 
Proof. By the assumption we have $\beta_{0}^{S}(\underline{b})=\operatorname{grade}_{S} S / \underline{b}$. Hence $\operatorname{grade}_{R} R / \underline{a} \geqq \beta_{0}^{R}(\underline{a})$ as $\operatorname{grade}_{R} R / \underline{a}=\operatorname{grade}_{S} S / \underline{b}$ by (6.1). This shows that $\underline{a}$ is generated by an $R$-regular sequence.

\section{REFERENCES}

[1] H. Bass, Algebraic K-theory, Benjamin, New York, 1968.

[2] C. Chevalley, Invariants of finite groups generated by reflexions, Amer. J. Math., 77 (1955), 778-782.

[ 3 ] C. W. Curtis and I. Reiner, Representation theory of finite groups and associative algebras, Pure and Applied Mathematics 11, Interscience Publishers, New York, 1962.

[4] S. Goto, When do the determinantal ideals define Gorenstein rings ?, Science Reports of the Tokyo Kyoiku Daigaku, Section A, 12 (1974), 129-145.

[5] - Invariant subrings under the action by a finite group generated by pseudoreflextions, Osaka J. Math., 15 (1978), 47-50.

[ 6 ] J. Herzog and E. Kunz, Der kanonische Modul eines Cohen-Macaulay-Rings, Lecture Notes in Mathematics 238, Springer.

[ 7 ] J.-P. Serre, Groupes finis d'automorphismes d'anneaux locaux réguliers, Colloq. d’Alg. E. N. S., 1967.

Department of Mathematics

Nihon University 